\title{
Our panel of experts highlight the most important research articles across the spectrum of topics relevant to the field of colorectal cancer
}

\author{
Expert panel: Franklin G Berger, María Dolores Giráldez, Antoni Castells \& Bruce D Minsky
}

Jovov B, Araujo-Perez F, Sigel CS et al. Differential gene expression between African American and European American colorectal cancer patients. PLOS ONE 7(1), e30168 (2012).

The disparately high incidence and mortality of colorectal cancer (CRC) in African-Americans is a major public health concern in the USA. Numerous studies have identified both socioeconomic as well as biologic factors as contributing to these disparities. In an attempt to assess the contribution of tumor gene expression to racial differences in CRC, Jovov et al. conducted a microarray analysis of sporadic cancers from 43 African-American and 43 European-American patients, using whole-genome cDNA arrays. They detected 95 genes that were expressed at different levels in the two racial groups. Expression of ten of these genes was validated by quantitative reverse transcriptase PCR in an additional 28 patients. A number of genes relating to inflammation and immune responses were identified among the differentially expressed genes, indicating these processes as potentially important physiological determinants of racial differences in disease aggressiveness and prognosis. In all, the results reported by Jovov et al. implicate differential gene expression as critical in CRC disparities between African-Americans and European-Americans, and complement other studies showing differences in frequencies of single nucleotide polymorphisms, in microsatellite instability, and in gene methylation patterns.

- Written by Franklin G Berger
Spindler KL, Pallisgaard N, Vogelius I, Jakobsen A. Quantitative cell-free DNA, KRAS and BRAF mutations in plasma from patients with metastatic colorectal cancer during treatment with cetuximab and irinotecan. Clin. Cancer Res. 18(4), 1177-1185 (2012).

The overall outcome of metastatic CRC has been improved by the use of monoclonal antibodies targeting the EGF receptor. However, these drugs are associated with a specific toxicity profile and are quite costly. Somatic KRAS mutations lead to resistance to this treatment, thus providing a useful strategy for tailoring such a treatment. However, tissue availability, tumor heterogeneity and mutational selection during disease progression represent important drawbacks of this approach. Cell-free DNA (cfDNA) in plasma is believed to originate mainly from tumor cells and could be tested for specific tumor mutations. This study analyzed the predictive and prognostic value of cfDNA, mutated $B R A F$ in plasma and mutated $K R A S$ in plasma (pmKRAS) at baseline in 108 patients with metastatic CRC in thirdline treatment with cetuximab and irinotecan. Only three patients harbored $B R A F$ mutations precluding further analysis. A high concordance between KRAS status in the primary tumor and plasma (plasma detection rate: $78 \%$; overall concordance: $91 \%$ ) was found. In addition, cfDNA in plasma and pmKRAS levels were strongly correlated (regression coefficient: 0.85; $\left.\mathrm{p}<10^{-4}\right)$. The disease control rate (i.e., percentage of patients who achieved complete or partial response, or stable disease) was significantly lower in patients with high
News \& Views

News

Journal Watch

Ask the Experts

Interviews
Future 
cfDNA ( $>75$ th percentile) than in those with low cfDNA ( $<25$ th percentile) (30 vs $77 \% ; \mathrm{p}=0.009)$. Moreover, all patients with pmKRAS levels above the 75th percentile suffered progressive disease (disease control rate: $0 \%$ ), whereas patients with pmKRAS below this percentile showed a disease control rate of $42 \%(\mathrm{p}=0.048)$. In a Cox regression analysis, high levels of cfDNA and/or pmKRAS were both strong predictors of a poor outcome. The authors concluded that circulating level of cfDNA and $\mathrm{pm} K R A S$ have potential value as biomarkers of response to treatment and clinical outcome in patients with metastatic CRC, providing a feasible alternative to tissue analysis.

- Written by María Dolores Giráldez $\&$ Antoni Castells

Alfa-Wali M, Annen-Mersh T, Antoniou $A$ et al. Chemoradiotherapy for anal cancer in HIV patients causes prolonged CD4 cell count suppression. Ann. Oncol. 23, 141-147 (2011).

Patients who are HIV-positive have a higher incidence of squamous cell cancer of the anus. Historically, they have received lower doses of radiation and chemotherapy due to the concern that standard therapy may not be tolerated. With the use of antiviral medications (highly active antiretroviral therapy), patients are able to better maintain their immune function. More recent reports have recommended that, in HIV-positive patients with a CD4 count $>200 \mathrm{~mm}^{-3}$ who do not have signs or symptoms of other HIV-related diseases, aggressive chemoradiation (CMT) is appropriate and results in a similar outcome to HIV-negative patients.

The report by Alfa-Wali et al. is a retrospective analysis of $60 \mathrm{HIV}$-positive patients with anal cancer treated over a 20 -year period. The median CD4 count was $305 \mathrm{~mm}^{-3}\left(16-1252 \mathrm{~mm}^{-3}\right)$. Overall, $83 \%$ of patients received CMT. The 5 -year disease-free survival was $76 \%$ and overall survival was $64 \%$. The most significant finding was independent of highly active antiretroviral therapy use, CMT resulted in prolonged CD4 count suppression. The post-treatment median CD4 count decreased from $289 \mathrm{~mm}^{-3}$ before CMT to $132 \mathrm{~mm}^{-3}$ at 3 months and $189 \mathrm{~mm}^{-3}$ at 1 year $(\mathrm{p}<0.05)$. By contrast, there was no change in the HIV viral load in those patients who received CMT plus highly active antiretroviral therapy. Of note, six patients who were without evidence of disease died due to AIDS-defining disease. Therefore, the authors concluded that prolonged CMT-induced CD4 suppression may have contributed to the late deaths.

- Written by Bruce D Minsky

\section{Financial \& competing interests disclosure}

The authors have no relevant affiliations or financial involvement with any organization or entity with a financial interest in or financial conflict with the subject matter or materials discussed in the manuscript. This includes employment, consultancies, honoraria, stock ownership or options, expert testimony, grants or patents received or pending, or royalties.

No writing assistance was utilized in the production of this manuscript.

\section{- Affiliations}

- Franklin G Berger

Center for Colon Cancer Research, Room 614, Jones Physical Sciences Center, University of South Carolina, Columbia, SC 29208, USA

- María Dolores Giráldez

Institute of Digestive \& Metabolic Diseases, Hospital Clinic, Villarroel 170, 08036 Barcelona, Catalonia, Spain

- Antoni Castells Institute of Digestive \& Metabolic Diseases, Hospital Clinic, Villarroel 170, 08036 Barcelona, Catalonia, Spain

- Bruce D Minsky

University of Chicago Hospitals, 5841 S Maryland Avenue, MC 2004, Chicago, IL 60637, USA 